EESTI NSV TEADUSTE AKADEEMIA TOIMETISED. XI KOIDE FOOSIKALIS-MATEMAATILISTE JA TEHNILISTE TEADUSTE SEERIA. 1962, NR. 2

ИЗВЕСТИЯ АКАДЕМИИ НАУК ЭСТОНСКОИ ССР. ТОМ ХІ

СЕРИЯ ФИЗИКО-МАТЕМАТИЧЕСКИХ И ТЕХНИЧЕСКИХ НАУК, 1962, No 2

\title{
ОБ ОЦЕНКЕ ПОГРЕШНОСТИ КУБАТУРНЫХ ФОРМУЛ
}

\section{М. ЛЕВИН}

О пределение 1. Функция $f(x, y)$ принадлежит классу $W^{(m, n)}(M, N, P)$, если на $K=[0 \leqslant x \leqslant 1,0 \leqslant y \leqslant 1]$ частные производные

$$
\frac{\partial^{i+j}}{\partial x^{i} \partial y^{j}} f(x, y) \quad(i=0,1, \ldots, m ; \quad j=0,1, \ldots, n)
$$

непрерывны и

$$
\left|\frac{\partial^{m}}{\partial x^{m}} f(x, y)\right| \leqslant M,\left|\frac{\partial^{n}}{\partial y^{n}} f(x, y)\right| \leqslant N,\left|\frac{\partial^{m+n}}{\partial x^{m} \partial y^{n}} f(x, y)\right| \leqslant P .
$$

Пусть мы имеем кубатурную формулу

$$
\int_{0}^{1} \int_{0}^{1} f(x, y) d x d y=\sum_{k=1}^{s} A_{k} f\left(x_{k}, y_{k}\right)+R[f],
$$

в которой $A_{k}, x_{k}, y_{k}(k=1,2, \ldots, s)$ выбраны так, что $R[f]=0$, если $f(x, y)$ многочлен относительно $x$ степени $\leqslant m-1$ и относительно $y$ степени $\leqslant n-1$. Следуя Крылову ['] и Никольскому $\left.{ }^{2}\right]$, найдем оценки для

$$
\begin{gathered}
R=\sup _{f^{\in W^{(m, n)}}} \mid R[\text { (M, N }, P)
\end{gathered}
$$

L1з (1) мы имеем квадратурные формулы

$$
\int_{0}^{1} g(x) d x \approx \sum_{k=1}^{s} A_{k} g\left(x_{k}\right) \quad \text { и } \int_{0}^{1} h(y) d y \approx \sum_{k=1}^{s} A_{k} h\left(y_{k}\right) \text {, }
$$

точные для многочленов степени $\leqslant m-1$ и $\leqslant n-1$ "соответственно.

Естественно считать известными величины

$$
\begin{aligned}
& r=\sup _{g \in W^{(m)}}|r[g(x)]|=\sup _{g \in W^{(m)}}\left|\int_{(P)}^{1} g(x) d x-\sum_{0}^{s} A_{k} g\left(x_{k}\right)\right|, \\
& \varrho=\sup _{h \in W_{(P)}^{(n)}}|\varrho[h(y)]|=\sup _{h \in W^{(n)}}\left|\int_{0}^{1} h(y) d y-\sum_{k=1}^{s} A_{k} h\left(y_{k}\right)\right|,
\end{aligned}
$$

где $W^{(l)}(P)-$ класс функций одной переменной, имеющих на $[0,1] l$ непрерывных производных, причем последняя ограничена по модулю числом $P$. 
Об оценке погрешности кубатурных формул

115

Т еорем а 1. Верно неравенство

$$
R \leqslant R_{1}+R_{2}+R_{3} \text {, }
$$

гde

$$
\begin{gathered}
R_{1}=\frac{\varrho}{(m+1) !}+M \sum_{k=1}^{s}\left|A_{k}\right| \int_{0}^{1}\left|\frac{(1-t)^{m}}{m !}-E\left(x_{k}-t\right) \frac{\left(x_{k}-t\right)^{m-1}}{(m-1) !}\right| d t \\
R_{2}=\frac{r}{(n+1) !}+N \sum_{k=1}^{s}\left|A_{k}\right| \int_{0}^{1}\left|\frac{(1-t)^{n}}{n !}-E\left(y_{k}-t\right) \frac{\left(y_{k}-t\right)^{n-1}}{(n-1) !}\right| d t, \\
R_{3}=P \int_{0}^{1} \int_{0}^{1} \mid \frac{(1-u)^{m}(1-t)^{n}}{m ! n !}-\sum_{k=1}^{s} A_{k} E\left(x_{k}-u\right) E\left(y_{k}-t\right) \frac{\left(x_{k}-u\right)^{m-1}\left(y_{k}-t\right)^{n-1} \mid d u d t}{(m-1) !(n-1) !} \\
E(x)= \begin{cases}1, & x>0 \\
0, & x \leqslant 0 .\end{cases}
\end{gathered}
$$

Доказательство. Представим $f(x, y)$ в форме Маклорена с остаточным членом в интегральной форме:

$$
\begin{gathered}
f(x, y)=\sum_{i=0}^{m-1} \frac{x^{i}}{i !} \frac{\partial^{i}}{\partial x^{i}} f(0, y)+\int_{0}^{x} \frac{\partial^{m}}{\partial x^{m}} f(t, y) \frac{(x-t)^{m-1}}{(m-1) !} d t= \\
=\sum_{i=0}^{m-1} \frac{x^{i}}{i !}\left[\sum_{k=0}^{n-1} \frac{y^{k}}{k !} \frac{\partial^{i+k}}{\partial x^{i} \partial y^{k}} f(0,0)+\int_{0}^{y} \frac{\partial^{i+n}}{\partial x^{i} \partial y^{n}} f(0, t) \frac{(y-t)^{n-1}}{(n-1) !} d t\right]+ \\
+\int_{0}^{x} \frac{\partial^{m}}{\partial x^{m}} f(t, y) \frac{(x-t)^{m-1}}{(m-1) !} d t .
\end{gathered}
$$

Обозначим

$$
P_{m-1, n-1}(x, y)=\sum_{i, k=0}^{m-1, n-1} \frac{x^{i} y^{k}}{i ! k !} \frac{\partial^{i+k}}{\partial x^{i} \partial y^{k}} f(0,0)
$$

Получаем

$$
\begin{gathered}
f(x, y)=P_{m-1, n-1}(x, y)+\int_{0}^{x} \frac{\partial^{m}}{\partial x^{m}} f(t, y) \frac{(x-t)^{m-1}}{(m-1) !} d t+ \\
+\int_{0}^{y} \frac{(y-t)^{n-1}}{(n-1) !} \sum_{i=0}^{m-1} \frac{x^{i}}{i !} \frac{\partial^{i+n}}{\partial x^{i} \partial y^{n}} f(0, t) d t= \\
=P_{m-1, n-1}(x, y)+\int_{0}^{x} \frac{\partial^{m}}{\partial x^{m}} f(t, y) \frac{(x-t)^{m-1}}{(m-1) !} d t+ \\
+\int_{0}^{y} \frac{(y-t)^{n-1}}{(n-1) !}\left[\frac{\partial^{n}}{\partial y^{n}} f(x, t)-\int_{0}^{x} \frac{\partial^{m+n}}{\partial x^{m} \partial y^{n}} f(u, t) \frac{(x-u)^{m-1}}{(m-1) !} d u\right] d t= \\
=P_{m-1, n-1}(x, y)+\varphi_{1}+\varphi_{2}+\varphi_{3},
\end{gathered}
$$


T де

$$
\begin{aligned}
\varphi_{1} & =\int_{0}^{x} \frac{\partial^{m}}{\partial x^{m}} f(t, y) \frac{(x-t)^{m-1}}{(m-1) !} d t=\int_{0}^{1} \frac{\partial^{m}}{\partial x^{m}} f(t, y) E(x-t) \frac{(x-t)^{m-1}}{(m-1) !} d t, \\
\varphi_{2} & =\int_{0}^{y} \frac{\partial^{n}}{\partial y^{n}} f(x, t) \frac{(y-t)^{n-1}}{(n-1) !} d t=\int_{0}^{1} \frac{\partial^{n}}{\partial y^{n}} f(x, t) E(y-t) \frac{(y-t)^{n-1}}{(n-1) !} d t, \\
\varphi_{3} & =-\int_{0}^{x} \int_{0}^{y} \frac{\partial^{m+n}}{\partial x^{m} \partial y^{n}} f(u, t) \frac{(x-u)^{m-1}(y-t)^{n-1}}{(m-1) !(n-1) !} d u d t= \\
& =-\int_{0}^{1} \int_{0}^{1} \frac{\partial^{m+n}}{\partial x^{m} \partial y^{n}} f(u, t) E(x-u) E(y-t) \frac{(x-u)^{m-1}(y-t)^{n-1}}{(m-1) !(n-1) !} d u d t .
\end{aligned}
$$

В силу определения (1)

$$
R\left[P_{m-1, n-1}\right]=0
$$

Поэтому

$$
R[f]=R\left[\varphi_{1}\right]+R\left[\varphi_{2}\right]+R\left[\varphi_{3}\right] .
$$

Оценим слагаемые $R\left[\varphi_{i}\right] \quad(i=1,2,3)$.

$$
R\left[\varphi_{1}\right]=\int_{0}^{1} d t R\left[\frac{\partial^{m}}{\partial x^{m}} f(t, y) E(x-t) \frac{(x-t)^{m-1}}{(m-1) !}\right]=
$$

$=\int_{0}^{1} d t\left[\int_{0}^{1} \int_{0}^{1} \frac{\partial^{m}}{\partial x^{m}} f(t, y) E(x-t) \frac{(x-t)^{m-1}}{(m-1) !} d x d y-\sum_{k=1}^{s} A_{k} \frac{\partial^{m}}{\partial x^{m}} f\left(t, y_{k}\right) E\left(x_{k}-t\right)\right.$.

$$
\begin{gathered}
\left.\frac{\left(x_{k}-t\right)^{m-1}}{(m-1) !}\right]=\int_{0}^{1} d t\left[\int_{0}^{1} \frac{\partial^{m}}{\partial x^{m}} f(t, y) d y \int_{t}^{1} \frac{(x-t)^{m-1}}{(m-1) !} d x-\right. \\
\left.-\sum_{k=1}^{s} A_{k} \frac{\partial^{m}}{\partial x^{m}} f\left(t, y_{k}\right) E\left(x_{k}-t\right) \frac{\left(x_{k}-t\right)^{m-1}}{(m-1) !}\right]= \\
\quad-\sum_{0}^{1} d t\left[\left(\sum_{k=1}^{s} A_{k} \frac{\partial^{m}}{\partial x^{m}} f\left(t, y_{k}\right)+\varrho\left[\frac{\partial^{m}}{\partial x^{m}} f(t, y)\right]\right) \frac{(1-t)^{m}}{m !}-\right. \\
\left.=\int_{0}^{1} \frac{(1-t)^{m}}{m !} \varrho\left[\frac{\partial^{m}}{\partial x^{m}} f(t, y)\right] d t+\int_{0}^{1} \sum_{k=1}^{s} A_{k} \frac{\partial^{m}}{\partial x^{m}} f\left(t, y_{k}\right) E\left(x_{k}-t\right) \frac{\left(x_{k}-t\right)^{m-1}}{(m-1) !}\right]= \\
\left.-E\left(x_{k}-t\right) \frac{\left(x_{k}-t\right)^{m-1}}{(m-1) !}\right] d t .
\end{gathered}
$$


Об оценке погрешности кубатурных формул

117

Отсюда

$$
\left|R\left[\varphi_{1}\right]\right| \leqslant R_{1}=\frac{\varrho}{(m+1) !}+M \sum_{k=1}^{s}\left|A_{k}\right| \int_{0}^{1}\left|\frac{(1-t)^{m}}{m !}-E\left(x_{k}-t\right) \frac{\left(x_{k}-t\right)^{m-1}}{(m-1) !}\right| d t
$$

Аналогично получаем

$$
\begin{gathered}
\left|R\left[\varphi_{2}\right]\right| \leqslant R_{2}=\frac{r}{(n+1) !}+N \sum_{k=1}^{s}\left|A_{k} ! \int_{0}^{1}\right| \frac{(1-t)^{n}}{n !}-E\left(y_{k}-t\right) \frac{\left(y_{k}-t\right)^{n-1}}{(n-1) !} \mid d t \\
\left|R\left[\varphi_{3}\right]\right| \leqslant R_{3}=P \int_{0}^{1} \int_{0}^{1} \mid \frac{(1-u)^{m}(1-t)^{n}}{m ! n !}- \\
-\sum_{k=1}^{s} A_{k} E\left(x_{k}-u\right) E\left(y_{k}-t\right) \frac{\left(x_{k}-u\right)^{m-1}\left(y_{k}-t\right)^{n-1}}{(m-1) !(n-1) !} \mid d u d t .
\end{gathered}
$$

Этим теорема доказана.

Оп ределение 2. Функция $f(x, y)$ принадлежит классу $\mathbb{W}_{0}^{(m, n)}(P)$, если на $K=[0 \leqslant x \leqslant 1,0 \leqslant y \leqslant 1]$ ее частные производные

$$
\frac{\partial^{i+j}}{\partial x^{i} \partial y^{j}} f(x, y) \quad(i=0,1, \ldots, m ; j=0,1, \ldots, n)
$$

непрерывны $и$

$$
\begin{aligned}
& \frac{\partial^{i}}{\partial x^{i}} f(0, y) \equiv \frac{\partial^{j}}{\partial y^{j}} f(x, 0) \equiv 0 \quad(i=0,1, \ldots, m-1 ; j=0,1, \ldots, n-1) . \\
&\left|\frac{\partial^{m+n}}{\partial x^{m} \partial y^{n}} f(x, y)\right| \leqslant P .
\end{aligned}
$$

Т еорем а 2. Пусть дана кубатурная формула

$$
\int_{0}^{1} \int_{0}^{1} f(x, y) d x d y \approx \sum_{k=0}^{r} A_{k} f\left(x_{k}, y_{k}\right)
$$

Тогда верна оценка

$$
\mid R\left[f(x, y]|=| \int_{0}^{1} \int_{0}^{1} f(x, y) d x d y-\sum_{k=0}^{r} A_{k} f\left(x_{k}, y_{k}\right)\left|\leqslant \frac{P}{(m-1) !(n-1) !} \int_{0}^{1} \int_{0}^{1} K_{m n}(t, u)\right| d t d: \cdot\right.
$$

гəิе

$$
K_{m n}(t, u)=\frac{(1-t)^{m}(1-u)^{n}}{m n}-\sum_{k=0}^{r} A_{k} E\left(x_{k}-t\right) E\left(y_{k}-u\right)\left(x_{k}-t\right)^{m-1}\left(y_{k}-u\right)^{n-1} .
$$

Доказательство. Используя формулу Маклорена с остаточным членом в интегральной форме, мы можем написать

$$
f(x, y)=\sum_{i=0}^{m-1} \frac{x^{i}}{i !} \frac{\partial^{i}}{\partial x^{i}} f(0, y)+\int_{0}^{x} \frac{\partial^{m}}{\partial x^{m}} f(t, y) \frac{(x-t)^{m-1}}{(m-1) !} d t .
$$


Учитывая (9) и используя функцию $E(x)$, получаем

$$
f(x, y)=\int_{0}^{1} \frac{\partial^{m}}{\partial x^{m}} f(t, y) E(x-t) \frac{(x-t)^{m-1}}{(m-1) !} d t .
$$

Аналогично находим

$$
\frac{\partial^{m}}{\partial x^{m}} f(t, y)=\int_{0}^{1} \frac{\partial^{m+n}}{\partial x^{m} \partial y^{n}} f(t, u) E(y-u) \frac{(y-u)^{n-1}}{(n-1) !} d u .
$$

Равенства (10) и (11) дают

$$
f(x, y)=\int_{0}^{1} \int_{0}^{1} \frac{\partial^{m+n}}{\partial x^{m} \partial y^{n}} f(t, u) E(x-t) E(y-u) \frac{(x-t)^{m-1}(y-u)^{n-1}}{(m-1) !(n-1) !} d t d u .
$$

Используя такое представление функции $f \in W_{0}^{(m, n)}(P)$, имеем

$$
\begin{gathered}
R[f(x, y)]=\int_{0}^{1} \int_{0}^{1} \frac{\partial^{m+n}}{\partial x^{m} \partial y^{n}} f(t, u) R\left[E(x-t) E(y-u) \frac{(x-t)^{m-1}(y-u)^{n-1}}{(m-1) !(n-1) !}\right] d t d u= \\
=\frac{1}{(m-1) !(n-1) !} \int_{0}^{1} \int_{0}^{1} \frac{\partial^{m+n}}{\partial x^{m} \partial y^{n}} f(t, u)\left[\int_{0}^{1} \int_{0}^{1} E(x-t) E(y-u)(x-t)^{m-1}(y-u)^{n-1} d x d y-\right. \\
\left.-\sum_{k=0}^{r} A_{k} E\left(x_{k}-t\right) E\left(y_{k}-u\right)\left(x_{k}-t\right)^{m-1}\left(y_{k}-u\right)^{n-1}\right] d t d u .
\end{gathered}
$$

Отсюда следует

$$
R[f(x, y)]=\frac{1}{(m-1) !(n-1) !} \int_{0}^{1} \int_{0}^{1} \frac{\partial^{m+n}}{\partial x^{m} \partial y^{n}} f(t, u) K_{m n}(t, u) d t d u,
$$

следовательно,

$$
|R[f(x, y)]| \leqslant \frac{P}{(m-1) !(n-1) !} \int_{0}^{1} \int_{0}^{1}\left|K_{m n}(t, u)\right| d t d u .
$$

Этим теорема доказана.

Оценка (12) точна для класса $W_{0}^{(m, n)}(P)$.

П р и м е р. Рассмотрим простейшую кубатурную формулу

$$
\int_{0}^{1} \int_{0}^{1} f(x, y) d x d y=f\left(\frac{1}{2}, \frac{1}{2}\right)+R[f]
$$

и найдем для нее оценку величины $R$. Здесь $m=n=2$. Вычисления по (3), (4), (5), (7) и (8) дают 


$$
\begin{gathered}
r=Q=P \int_{0}^{1}\left|\frac{(1-t)^{2}}{2}-E\left(\frac{1}{2}-t\right)\left(\frac{1}{2}-t\right)\right| d t=\frac{P}{24}, \\
R_{1}=\frac{P}{144}+\frac{M}{24}, \quad R_{2}=\frac{P}{144}+\frac{N}{24} \text { и } R_{3}=\frac{7 P}{576},
\end{gathered}
$$

отхуда

$$
R \leqslant \frac{M+N}{24}+\frac{5 P}{192}
$$

\section{Л И Т Е Р А Т Р А}

1. К рылов В. И., Приближенное вычисление интегралов. Москва, 1959.

2. Ни кольски й С. М., Қвадратурные формулы. Москва, 1958.

Ннститит кибернетики

Академии наук Эстонской ССР

\section{Поступила в редакцию}

13. XII 1961

\section{KUBATUURVALEMITE VEA HINNANGUST}

\section{Levin}

\section{Resümee}

Artiklis esitatakse kubatuurvalemi (1) vea ülemine tõke diferentseeruvate funktsioonideklassi $\mathbb{W}(m, n)(M, N, P)$ jaoks.

\section{Eesti NSV Teaduste Akadeemia Küberneetika Instituut}

\section{Saabus toimetusse}

13. XII 1961

\section{ON THE ESTIMATE OF THE ERROR FOR QUADRATURE IN TWO DIMENSIONS}

\section{Levin \\ Summary}

The present article deduces the upper boundary of the errors of the quadrature formula (1) for the function class $W(m, n)(M, N, P)$ which is subject to differentiation.

Academy of Sciences of the Estonian S.S.R.,

Received Institute of Cybernetics 